\title{
Vecteurs rétroviraux pour le transfert de gènes dans le tissu hématopoiétique in vivo
}

Le transfert de gènes dans les cellules hématopoïétiques pourrait être indiqué pour traiter des maladies moléculaires de l'hémoglobine, des déficits immunologiques par anomalie enzymatique, voire pourrait être intégré à des stratégies de traitement des cancers solides et des leucémies. Les vecteurs rétroviraux permettent d'espérer un transfert très efficace des gènes $d$ 'intérêt. Le perfectionnement constant des vecteurs et les connaissances accumulées sur les propriétés des cellules de la moelle osseuse, principalement les cellules souches, ont permis d'obtenir récemment, chez l'animal d'expérience, des expressions stables et relativement fortes de plusieurs types de gènes intégrés dans les cellules médullaires.

\section{Pierre Lehn}

P. Lehn : maître de conférences, université Paris$V$ (CHU Paris-Ouest). Praticien hospitalier, unité de greffe de moelle, département d'hématologie, hôpital Saint-Louis, 1, avenue Claude-Vellefaux, 75475 Paris Cedex 10, France.

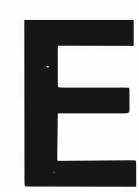
n complément du dossier "Bio-éthique et génétique " rapportant les problèmes discutés lors du colloque "Patrimoine génétique et droits de l'humanité ", les perspectives actuelles de thérapie génique ont été discutées par A. Kahn et P. Briand dans médecine/sciences $1990, n^{\circ} 2$, vol. 6 , p. 125-51. Les diverses approches de thérapie génique ont alors été envisagées de façon très synthétique. Notre but, ici, est de discuter plus en détail l'utilisation de vecteurs rétroviraux pour le transfert de gènes dans le tissu hématopoiétique in vivo, puisque la majorité des études chez l'animal ont concerné cette approche de thérapie génique somatique. Nous ferons donc le point sur les aspects techniques de la production et de l'utilisation des vecteurs rétroviraux, ainsi que sur les résultats obtenus à ce jour. Certes, des difficultés persistent, mais des solutions pour les résoudre sont maintenant en vue.
Enfin, diverses indications potentielles de thérapie génique en oncohématologie seront présentées.

\section{Thérapie génique} somatique " additive "

Alors que la thérapeutique conventionnelle utilise des substances capables d'interférer à divers niveaux avec le fonctionnement de la cellule, les récents progrès de la génétique moléculaire permettent d'envisager d'agir au niveau du génome luimême. Une importante voie de recherche consiste ainsi en la mise au point de substances interagissant avec des séquences nucléotidiques spécifiques dans le but de modifier l'expression de gènes bien précis. On peut aussi envisager d'utiliser le matériel génétique lui-même dans un but thérapeutique : c'est la thérapie génique par transfert de gènes.

C'est en premier lieu aux anomalies héréditaires récessives dont le traitement ne nécessite que l'addition d'un 


\section{RÉFÉRENCES}

1. Lehn PM. Gene therapy using bone marrow transplantation. Bone Marrow Transplant 1987 ; 1 : 243-58.

2. Lehn PM. Gene therapy using bone marrow transplantation : a 1990 update. Bone Marrow Transplant 1990 ; 5 : 287-93.

3. Anderson WF. Prospects for human gene therapy. Science $1984 ; 226$ : 401-9.

4. Hershfield MS, Buckley RH, Greenberg ML, et al. Treatment of adenosine deaminase deficiency with polyethylene glycolmodified adenosine deaminase. $N$ Engl J Med 1987 ; 316 : 589-96.

5. Barton NW, Furbish FS, Murray GJ, Garfield M, Brady RO. Therapeutic response to intravenous infusions of glucocerebrosidase in a patient with Gaucher disease. Proc Natl Acad Sci USA 1990 ; 87 : 1913-6.

6. Weiss R, Teich N, Varmus $\mathrm{H}$, Coffin $\mathrm{J}$. RNA Tumor Viruses. Cold Spring Harbor, New York (USA): Cold Spring Harbor Laboratory, 1984.

7. Temin HM. Retrovirus vectors for gene transfer. In : Kucherlapati, ed. Gene Transfer. New York: Plenum Press, 1986 : 149-87.

8. Prats AC, Sarih L, Gabus C, Litvak S, Keith G, Darlix JL. Small finger protein of avian and murine retroviruses has nucleic acid annealing activity and positions the replication primer tRNA onto genomic RNA. $E M B O J 1988 ; 7: 1777-83$

9. Mann R, Mulligan RC, Baltimore D. Construction of a retrovirus packaging mutant and its use to produce helper-free defective retrovirus. Cell 1983 ; 33 : 153-9.

10. Miller AD, Buttimore C. Redesign of retrovirus packaging cell lines to avoid recombination leading to helper virus production. Mol Cell Biol 1986 ; 6 : 2895-02.

11. Markowitz D, Goff S, Bank A. A safe packaging line for gene transfer : separating viral genes on two different plasmids. $J$ Virol $1988 ; 62: 1120-4$

12. Danos O, Mulligan RC. Safe and efficient generation of recombinant retroviruses with amphotropic and ecotropic host ranges. Proc Natl Acad Sci USA 1988 ; 85 : 6460-4.

13. Cone RD, Mulligan RC. Highefficiency gene transfer into mammalian cells : generation of helper-free recombinant retroviruses with broad mammalian host range. Proc Natl Acad Sci USA 1984; 81 : 6349-53.

14. Spangrude GJ, Heimfeld S, Weissman IL. Purification and characterization of mouse hematopoietic stem cells. Science $1988 ; 241: 58-62$ gène normal que s'adresse ce type de thérapie. Cependant, la possibilité par recombinaison homologue de remplacer un gène muté par un gène normal n'exclut pas les maladies dominantes de cette approche thérapeutique. Évidemment, l'anomalie génétique en cause doit être connue et il faut pouvoir disposer de la version normale du gène en question, qui doit donc avoir été cloné.

Il faut, en outre, s'assurer que le transfert de gènes n'atteint pas les cellules de la lignée germinale. Il est clair qu'un gène spécifiquement transféré dans le tissu hématopoïétique, uniquement constitué de cellules somatiques, ne sera pas transmis à la descendance. Encore faut-il s'assurer qu'une fois transféré dans ce tissu, il ne peut, grâce à son vecteur viral, être transporté vers d'autres cellules, en particulier de la lignée germinale. Le caractère défectif des vecteurs rétroviraux utilisés et leur mode de production rendent très improbable un tel événement. Sur le plan éthique, il ne devrait donc pas y avoir de problèmes particuliers, puisque le tissu germinal n'est pas modifié. Cependant, comme pour toute nouvelle thérapeutique, la thérapie génique somatique devra faire la preuve de son efficacité et devra être comparée aux autres traitements déjà existants, notamment à la greffe de moelle osseuse allogénique [1-3]. Enfin, bien que l'administration répétée d'enzymes purifiées puisse également être utile pour le traitement de certaines maladies génétiques récessives, il faut insister sur le fait que la thérapie génique, tout comme la greffe de moelle osseuse allogénique, vise à constituer un traitement définitif $[4,5]$.

\section{La moelle osseuse pour cible}

La majorité des expériences faites jusqu'à présent chez l'animal ont concerné le transfert de gènes dans la moelle osseuse. En effet, la moelle est relativement facile à manipuler, qu'il s'agisse du prélèvement, du transfert de gènes ex vivo ou de l'autogreffe par réinjection intraveineuse de la moelle génétiquement modifiée. L'animal " receveur " est habituellement irradié afin de détruire son hématopoïèse endogène et d'obtenir la reconstitution hématologique à partir de la moelle génétiquement manipulée. Si l'on parvenait à ce que le transfert de gènes puisse conférer un avantage de croissance aux cellules, l'ablation complète de l'hématopoïèse endogène ne serait peut-être même plus nécessaire.

Une donnée essentielle est que la moelle osseuse contient les cellules souches hématopoïétiques (CSH) capables tout à la fois de se renouveler et d'assurer une hématopoïèse complète qui sera permanente après greffe. Ce sont ces CSH qui sont les cellules cibles pour le transfert de gènes. En effet, un gène transféré dans les $\mathrm{CSH}$ va donc persister de façon stable dans le tissu hématopoïétique, permettant ainsi d'envisager un traitement définitif de l'anomalie génétique. Par ailleurs, le gène transféré dans les $\mathrm{CSH}$ va être transmis aux cellules différenciées qui en dérivent. La correction d'un déficit génétique étant liée à l'expression du transgène dans les cellules différenciées adéquates, il est donc essentiel que le provirus transféré contienne les séquences régulatrices lui permettant d'être fonctionnel dans les cellules où il doit assurer sa fonction. Bref, si la moelle osseuse semble un tissu cible intéressant en raison de certaines facilités techniques pour sa manipulation, sa biologie fort complexe rend délicate l'approche " transfert de gènes dans les CSH ".

\section{Principales étapes du cycle rétroviral}

Pour pouvoir comprendre l'intérêt (et les inconvénients) des vecteurs rétroviraux, il est nécessaire d'avoir à l'esprit les principales étapes du cycle réplicatif d'un rétrovirus $[6,7]$. Il s'agit de virus dont le génome est constitué d'un dimère d'ARN [8]. Leur capside est entourée par une enveloppe permettant l'adhérence à la membrane cellulaire. Les virions contiennent également une enzyme particulière, la transcriptase inverse. Le génome viral comporte les trois gènes gag, pol et env qui codent respectivement pour les protéines de la capside, pour la transcriptase inverse et pour les protéines de l'enveloppe. De plus, le génome viral comporte 
des séquences nécessaires en cis durant le cycle viral.

Après pénétration du virus dans la cellule, l'ARN viral sert de matrice pour la production d'une copie ADN double brin par transcription inverse. L'ADN va alors s'intégrer au hasard dans le génome cellulaire, en $y$ créant un "provirus " ayant à chaque extrémité une séquence identique appelée longue répétition terminale (long terminal repeat, LTR). La transcription du provirus intégré est sous la dépendance de signaux transcriptionnels situés dans le LTR 5'. Les protéines Gag et Pol sont obtenues par traduction d'ARN génomique néoformé alors que Env provient de la traduction d'un messager sousgénomique obtenu par épissage. De plus, une fraction des molécules d'ARN génomique néoformées n'est pas traduite et va être encapsidée dans les nouveaux virions. L'encapsidation se fait par interaction entre une séquence particulière du génome viral (séquence Psi) et certaines protéines de structure du rétrovirus. Finalement, le virus bourgeonne à la surface cellulaire.

Schématiquement, dans un vecteur rétroviral, les gènes gag, pol et env ont été ôtés et sont remplacés par le(s) gène(s) à transférer, alors que les séquences nécessaires en cis (notamment la séquence Psi) sont conservées [7]. La plupart des vecteurs construits jusqu'à présent dérivent du génome du virus de la leucémie murine de Moloney (Mo-MuLV).

\section{Cellules d'encapsidation}

L'emploi de " cellules d'encapsidation" a pour but l'obtention de stocks de rétrovirus vecteurs ne contenant pas de virus sauvages auxiliaires (helper) capables de se propager (stocks "helper-free ") (figure 1). Il s'agit de fibroblastes 3T3 dans le génome desquels on a introduit par transfection un provirus Mo-MuLV débarrassé de la séquence d'encapsidation Psi $[9,10]$. Ce provirus Moloney est donc capable de diriger la synthèse de l'ensemble des constituants du virus, mais l'ARN génomique sans séquence Psi (Psi -) ne peut pas être encapsidé efficacement. Lorsque l'on introduit dans ces cellules un vecteur rétroviral Psi + , il se $\mathrm{m} / \mathrm{s} n^{\circ} 8$, vol. 6 , octobre 90

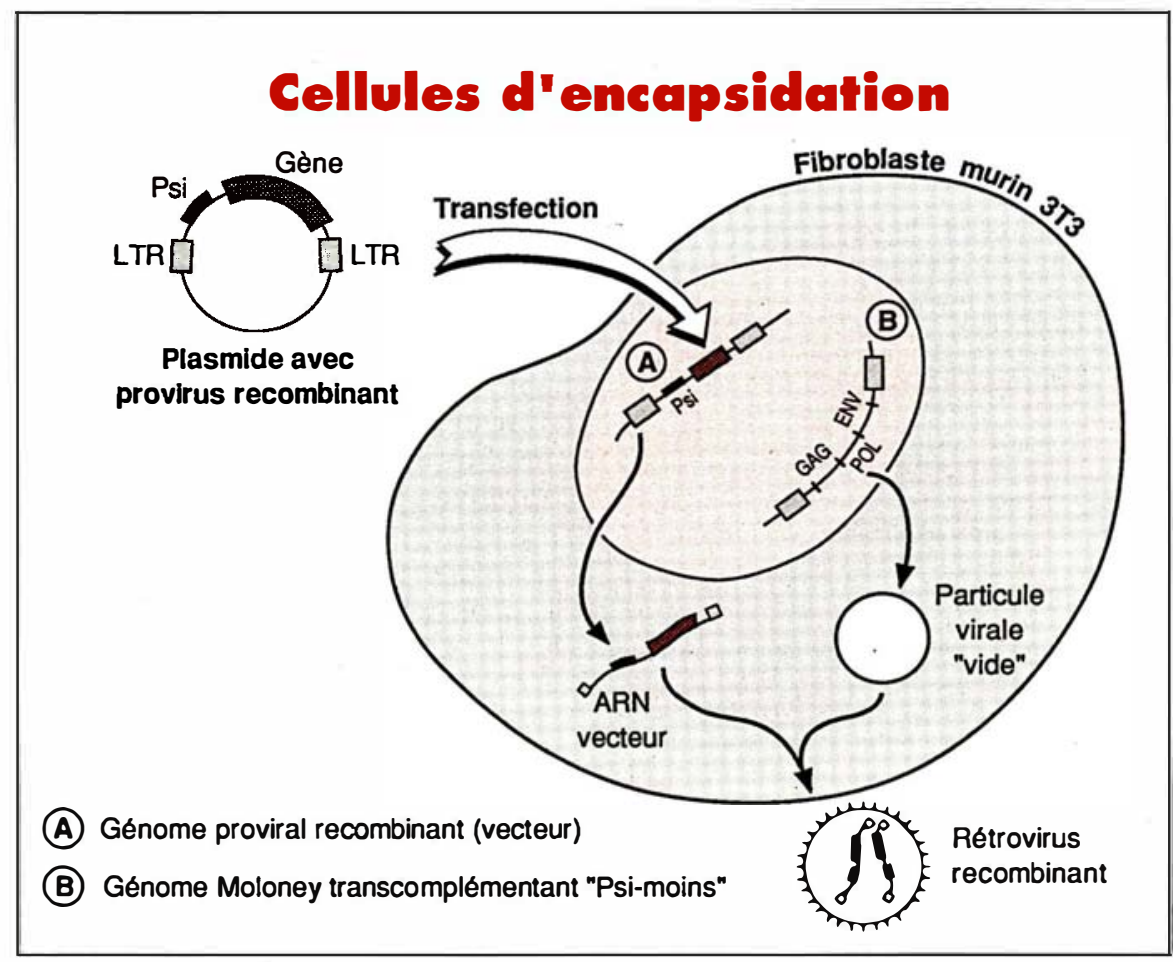

Figure 1. Cellules d'encapsidation. Le schéma explique le principe de leur emploi pour obtenir des stocks de rétrovirus vecteurs ne contenant pas de virus " helpers" capables de se propager. En effet, un vecteur rétroviral Psiplus est encapsidé dans la particule virale produite par le provirus Moloney Psi-moins.

produit alors une " transcomplémentation ": le vecteur recombinant Psi + va pouvoir être encapsidé dans la particule virale produite par le provirus Moloney Psi - . Il y a donc production de stocks de rétrovirus recombinants ne contenant pas de virus helper. Ces rétrovirus vecteurs sont capables d'infecter les cellules cibles et d'y intégrer leur génome recombinant, mais ne peuvent se propager plus loin (single hit). Dans le cas de cellules médullaires transduites ex vivo puis réinjectées à l'animal d'expérience, il n'y a donc pas de propagation du vecteur au-delà du tissu hématopoïétique et donc en particulier aux cellules germinales.

Récemment, la fiabilité des cellules d'encapsidation a été améliorée par l'introduction de modifications supplémentaires dans le génome Moloney transcomplémentant, afin de diminuer le risque de formation de virus sauvage par recombinaison avec le vecteur rétroviral [11, 12]. On dispose donc à présent de lignées d'encapsidation fiables pour produire des stocks de rétrovirus vecteurs soit écotropes (spécifiques des cellules murines), soit amphotropes (à tropisme plus étendu incluant les cellules humaines). Pour la production de vecteurs amphotropes, le gène env Moloney du génome transcomplémentant est remplacé par le gène env $\mathrm{du}$ virus murin amphotrope 4070 A [13].

\section{Vecteurs rétroviraux : principaux avantages}

Comme tous les virus, les rétrovirus recombinants sont capables d'infecter simultanément un grand nombre de cellules. Cela est particulièrement important dans le cas de la moelle osseuse, où les $\mathrm{CSH}$ sont très rares (environ 1 sur 10000 cellules). De plus, l'utilisation de techniques permettant de concentrer (voire de purifier) les $\mathrm{CSH}$ pourrait permettre de favoriser leur infection [14, 15]. 
15. Szilvassy SJ, Fraser CC, Eaves CJ, Lansdorp PM, Eaves AC, Humphries RK. Retrovirus-mediated gene transfer to purified hemopoietic stem cells with long-term lympho-myelopoietic repopulating ability. Proc Natl Acad Sci USA 1989 ; 86 : 8798-02.

16. Albritton LM, Tseng L, Scadden D, Cunningham JM. A putative murine ecotropic retrovirus receptor gene encodes a multiple membrane-spanning protein and confers susceptibility to virus infection. Cell 1989 ; 57 : 659-66.

17. Keller G, Paige C, Gilboa E, Wagner EF. Expression of a foreign gene in myeloïd and lymphoïd cells derived from multipotent haematopoietic precursors. Nature $1985 ; 318$ : 149-54.

18. Dick JE, Magli MC, Huszar D, Phillips RA, Bernstein A. Introduction of a selectable gene into primitive stem cells capable of long-term reconstitution of the hematopoietic system of W/Wc mice. Cell 1985 ; 42 : 71-9.

19. Lemischka IR, Raulet DH, Mulligan RC. Developmental potential and dynamic behavior of hematopoietic stem cells. Cell $1986 ; 45$ : 917-27.

20. Schuening FG, Storb R, Stead RB, Goehle S, Nash R, Miller AD. Improved retroviral transfer of genes into canine hematopoietic progenitor cells kept in longterm marrow culture. Blood 1989; 74 : $152-5$

21. Hock RA, Miller AD. Retrovirusmediated transfer and expression of drug resistance genes in human hematopoietic progenitor cells. Nature $1986 ; 320$ : 27.5-7.

22. Roux P, Jeanteur P, Piechaczyk M. A versatile and potentially general approach to the targetting of specific cell types by retroviruses. Proc Natl Acad Sci USA 1989 ; 86 : 9079-83.

23. Lim B, Apperley JF, Orkin SH, Williams DA. Long-term expression of human $A D A$ in mice transplanted with retrovirus infected hematopoietic stem cells. Proc Natl Acad Sci USA 1989 ; 86 : 8892-6.

24. Bodine DM, Karlsson S, Nienhuis AW. Combination of interleukins 3 and 6 preserves stem cell function in culture and enhances retrovirus-mediated gene transfer into hematopoietic stem cells. Proc Natl Acad Sci USA 1989 : 86 : 8897-01.

25. Al-Lebban ZS, Henry JM, Jones JB, Eglitis MA, Anderson WF, Lothrop CO. Increased efficiency of gene transfer with retroviral vectors in neonatal hematopoietic progenitor cells. Exp Hematol $1990 ; 18$ : 180-4.
Surtout, les vecteurs rétroviraux ont la capacité de s'intégrer très efficacement dans le génome de la cellule infectée. Le gène transféré va donc persister de façon stable dans le génome de la CSH infectée et dans les cellules différenciées qui en dérivent. De plus, le vecteur s'intègre sans entraîner de remaniements importants du génome de la cellule cible et le provirus formé a une structure bien définie, prévisible, respectant la structure du gène transféré.

\section{Inconvénients des vecteurs rétroviraux}

Tout d'abord, la taille du gène pouvant être transporté par un vecteur rétroviral est limitée à environ $8 \mathrm{~kb}$ [7]. Pour des gènes trop grands, on est donc obligé de transférer leur $\mathrm{ADN}_{\mathrm{C}}$ et il faut alors construire un minigène fonctionnel.

En ce qui concerne l'interaction entre l'enveloppe du rétrovirus et son récepteur cellulaire, c'est seulement récemment que le récepteur des virus écotropes a été cloné, alors que les études in vivo avaient déjà montré que les $\mathrm{CSH}$ murines pouvaient être infectées par des vecteurs rétroviraux [16-19]. Au contraire, le récepteur des virus amphotropes est toujours inconnu. Néanmoins, les progéniteurs hématopoïétiques de diverses espèces (chien, chat, singe...), y compris l'homme, étant infectables par des rétrovirus amphotropes, il est probable que le récepteur amphotrope soit aussi présent sur les $\mathrm{CSH}$ humaines [20, 21]. Il se pourrait cependant que des rétrovirus non murins (notamment simiens) soient plus efficaces pour infecter les CSH humaines. Finalement, l'utilisation d'anticorps pour constituer des ponts bifonctionnels (qui reconnaissent à la fois la glycoprotéine Env du virus et un marqueur de la surface cellulaire) pourrait se révéler très intéressante pour cibler les rétrovirus vecteurs au niveau des CSH [22].

L'intégration d'un rétrovirus dans le génome de la cellule cible requiert que celle-ci soit en division. Comme la très grande majorité des $\mathrm{CSH}$ sont en Go, c'est-à-dire dans un état quiescent, il faut donc les mettre en cycle, sans cependant leur faire per- dre leurs propriétés de cellules souches (autorenouvellement, totipotentialité). Diverses méthodes ont été utilisées dans ce but : prétraitement des souris donneuses par du 5-fluorouracile (5-FU), étape de "préstimulation " de la moelle in vitro avant l'infection, utilisation de combinaisons adéquates de facteurs de croissance (IL 3 et IL 6) dans le milieu d'infection [18, 23, 24]. Un apport important de la biologie cellulaire serait de définir les conditions optimales pour l'infection des CSH. Il se pourrait cependant que des $\mathrm{CSH}$, à un autre stade du développement (notamment des cellules fotales ou néonatales), constituent des cibles plus faciles [25].

Il est classiquement admis que l'intégration d'un rétrovirus se fait au hasard dans le génome de la cellule cible. Il semble cependant que l'intégration ait lieu préférentiellement dans les régions actives, " ouvertes" de l'ADN, caractérisées par leur hypersensibilité à la désoxyribonucléase 1 . Le but de la thérapie génique étant le transfert d'un gène donné dans les $\mathrm{CSH}$ et son expression dans les cellules différenciées adéquates, l'utilisation de vecteurs rétroviraux rencontre ici une double difficulté : intégration du vecteur dans une région d'ADN active dans la $\mathrm{CSH}$ mais devenant inactive lors de la différenciation ou intégration dans une région d'ADN inactive dans la $\mathrm{CSH}$, avec mise en jeu de mécanismes d'inactivation rendant le provirus définitivement inactif. Ces considérations imposent la mise au point de vecteurs transportant un véritable "minilocus" capable de fonctionner de façon adéquate indépendamment du site d'intégration. Par ailleurs, l'intégration "au hasard " du vecteur peut aboutir à une mutagenèse d'insertion et présente le risque d'activer un protooncogène cellulaire proche du site d'intégration [7]. Bien qu'il n'y ait pas eu d'étude spécifique de ce point, le risque de cancérogenèse apparaît néanmoins très faible dans l'expérience actuelle de divers groupes de recherches utilisant des vecteurs rétroviraux. En effet, à la différence des rétrovirus naturels, les rétrovirus utilisés dans un but de thérapie génique sont incapables de se propager 
et ne transportent pas d'oncogène. Ces considérations invitent quand même à réserver une éventuelle thérapie génique par des rétrovirus recombinants au traitement de maladies très graves.

\section{Les divers types de vecteurs rétroviraux}

Les vecteurs rétroviraux peuvent être classés en fonction de la nature et du nombre des séquences codantes trans- portées (fragment génomique ou $\mathrm{ADN}_{\mathrm{c}}$, présence ou non d'un gène de sélection) et aussi en fonction des signaux transcriptionnels utilisés (signaux rétroviraux du LTR 5' ou éléments transcriptionnels hétérologues rajoutés dans le vecteur) [7] (figure 2). Il faut souligner qu'il n'y a vraisemblablement pas de vecteur " universel " utilisable pour n'importe quel gène. Il en découle que chaque vecteur doit être testé empiriquement.
Les premiers vecteurs construits utilisaient les signaux transcriptionnels du LTR Moloney. Les études initiales in vivo ont alors suggéré que ces vecteurs ne permettaient pas une expression adéquate du gène transféré après greffe du tissu hématopoïétique [17]. On s'est donc orienté vers l'emploi d'autres signaux transcriptionnels. D'une part, le LTR peut être modifié : le enhancer du LTR Moloney peut être remplacé par des séquences enhancers hétérologues. Il

\section{Divers types de vecteurs rétroviraux}

1 Provirus Moloney :

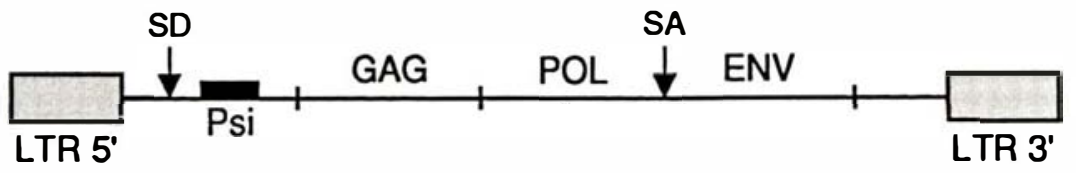

2. ARN de Moloney :

a/ ARN génomique

b/ ARN sous-génomique

3. Vecteur à LTR :

4. Vecteur à promoteur interne (P. INT.) :

5. Vecteur SIN :
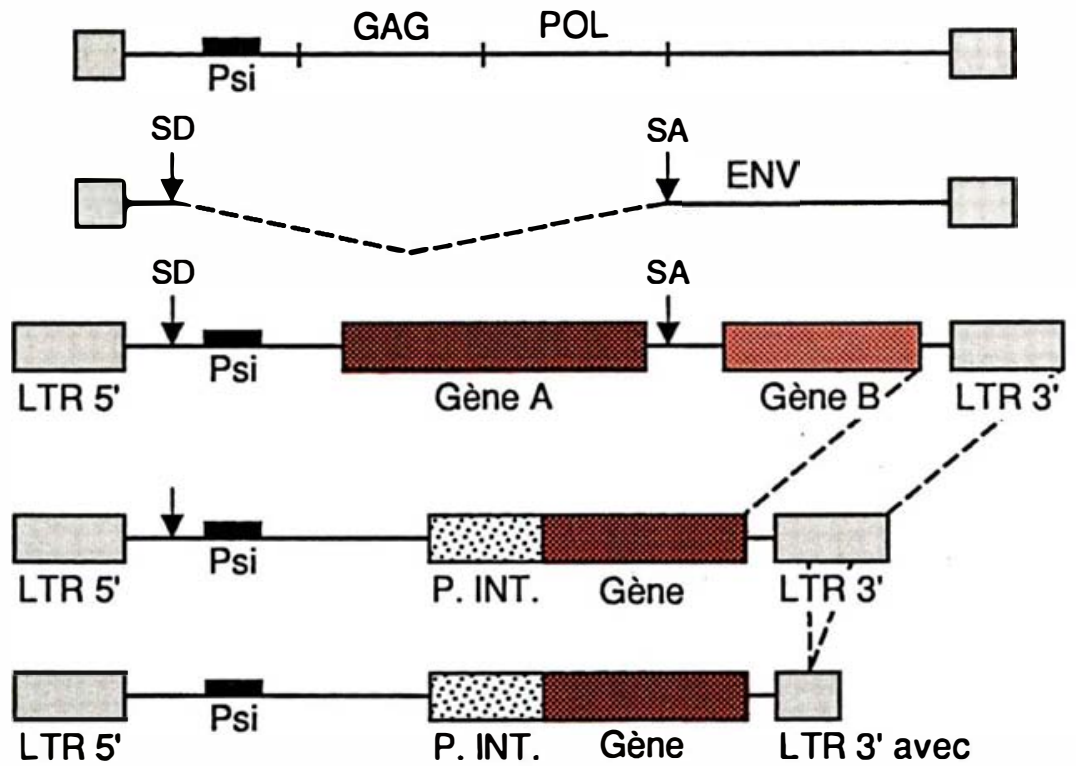

LTR 3 ' avec délétion

Figure 2. Représentation schématique de la structure provirale du virus de la leucémie murine de Moloney et des divers types de vecteurs rétroviraux. 1. Le schéma du provirus Moloney indique les gènes gag, pol, env et la séquence Psi pour l'encapsidation. Les sites donneur et accepteur d'épissage sont également indiqués. 2. La transcription du provirus Moloney est sous la dépendance des signaux transcriptionnels du LTR $5^{\prime}$. Elle aboutit à un ARN génomique (pour les protéines Gag et Pol) et, après épissage, à un ARN sous-génomique (pour les protéines Env). 3. Dans un vecteur à $L T R$, les gènes $A$ et $B$, qui remplacent respectivement les séquences gal-pol et env, sont sous le contrôle transcriptionnel du LTR 5'. La protéine B est obtenue à partir du messager épissé. 4 . Dans un vecteur à promoteur interne, la transcription du gène d'intérêt est sous le contrôle de signaux transcriptionnels hétérologues incorporés dans l'unité transcriptionnelle rétrovirale. 5. Dans un vecteur SIN (self inactivating), les séquences enhancers du LTR $3^{\prime}$ sont délétées. La transcription inverse dans les cellules cibles aboutit à un provirus auquel manquent les séquences enhancers rétrovirales au niveau des 2 LTRs. La transcription du gène d'intérêt repose alors sur le promoteur interne. 
RÉFÉRENCES

26. Robbins PD, Lehn PM, Mulligan RC. Regulation of retroviral transcription in embryonic carcinoma cells and in hematopoietic cells in vivo. Cold Spring Harbor Symp. "Mouse molecular genetics " $\mathrm{CSH}$ New York $1988 ; 74$.

27. Yu SF, Von Ruden $T$, Kantoff PW, et al. Self-inactivating retroviral vectors designed for transfer of whole genes into mammalian cells. Proc Natl Acad Sci USA 1986 ; 83 : 3194-8.

28. Cone RD, Weber-Benarous A, Baorto $D$, Mulligan RC. Regulated expression of a complete human $\beta$-globin gene encoded by a transmissible retrovirus vector. $\mathrm{Mol} \mathrm{Cell}$ Biol 1987 ; 7 : 887-97.

29. Hantzopoulos PA, Sullenger BA, Ungers G, Gilboa E. Improved gene expression upon transfer of the adenosine deaminase minigene outside the transcriptional unit of a retroviral vector. Proc Natl Acad Sci USA 1989 ; 86 : 3519-23.

30. Lehn PM, Robbins PD, Mulligan RC. Retroviral vectors for the transfer of a mutated DHFR* cDNA into mouse bone marrow cells in vivo. $J$ Cell Biochemistry 1988 : suppl 12B : 179 .

31. Dzierzak EA, Papayannopoulou T, Mulligan RC. Lineage-specific expression of a human $\beta$-globin gene in murine bone marrow transplant recipients reconstituted with retrovirus transduced stem cells. Nature $1988: 331: 35-41$

32. Bender MA, Gelinas RE, Miller AD. A majority of mice show long-term expression of a human $\beta$-globin gene af ter retrovirus transfer into hematopoietic stem cells. Mol Cell Biol 1989 ; 9 : 1426-34

33. Jordan CT, Lemischka IR. Clonal and systemic analysis of long-term hematopoiesis in the mouse. Genes Dev 1990; 4 : 220-32.

34. Bowtell DDL, Johnson GR, Kelso A, Cory S. Expression of genes transferred to hematopoietic stem cells by recombinant retroviruses. Mol Biol Med 1987 ; 4 : 229-50.

35. Guild BC, Finer HM, Housman DE, Mulligan RC. Development of retrovirus vectors useful for expressing genes in cultured murine embryonal cells and hematopoietic cells in vivo. J Virol 1988; 62 : 3795-01.

36. Williams DA, Hsieh K, De Silva A, Mulligan RC. Protection of bone marrow transplant recipients from lethal doses of methotrexate by the generation of methotrexate-resistant bone marrow. $J$ Exp s'agit alors de LTRs substitués, hybrides. Ainsi, divers travaux avec des cellules indifférenciées de carcinome embryonnaire (où le LTR Moloney est inactif) suggèrent d'utiliser les séquences enhancers du rétrovirus murin MPSV (myeloproliferative sarcoma virus) ou de mutants du virus Polyome, car les CSH sont également des cellules peu différenciées [26].

D'autre part, des signaux transcriptionnels "puissants" peuvent être rajoutés aux vecteurs rétroviraux. Dans ces vecteurs à "promoteur interne ", l'expression d'un $\mathrm{ADN}_{\mathrm{c}}$ transféré est alors sous la dépendance d'un promoteur hétérologue (d'origine virale ou cellulaire) ou d'une combinaison enhancer-promoteur complexe incorporée à l'unité transcriptionnelle du rétrovirus. Ces vecteurs devraient posséder la flexibilité requise pour obtenir une expression relativement adéquate du gène transféré. Une modification supplémen- taire consiste à déléter les séquences transcriptionnelles rétrovirales du LTR qui sont la cible éventuelle d'un processus d'inactivation (selfinactivating SIN vectors) [27, 28]. Toujours dans le but de protéger la transcription du transgène des interférences avec le LTR rétroviral, on a également suggéré l'emploi de vecteurs dits "double copie ", dans lesquels le gène à transférer est incorporé dans le LTR du vecteur en dehors de l'unité transcriptionnelle rétrovirale [29].

Le gène $\beta$-globine constitue un important cas particulier. En effet, en raison de la petite taille du gène de structure $\beta$-globine, il est possible d'insérer dans le vecteur un véritable fragment génomique comportant exons, introns et séquences régulatrices flanquantes en 5' et en 3' (vecteur génomique) [28]. Nous verrons plus loin comment une meilleure connaissance du fonctionnement du locus $\beta$-globine permet à présent

\section{Etapes du transfert de gènes chez la souris}

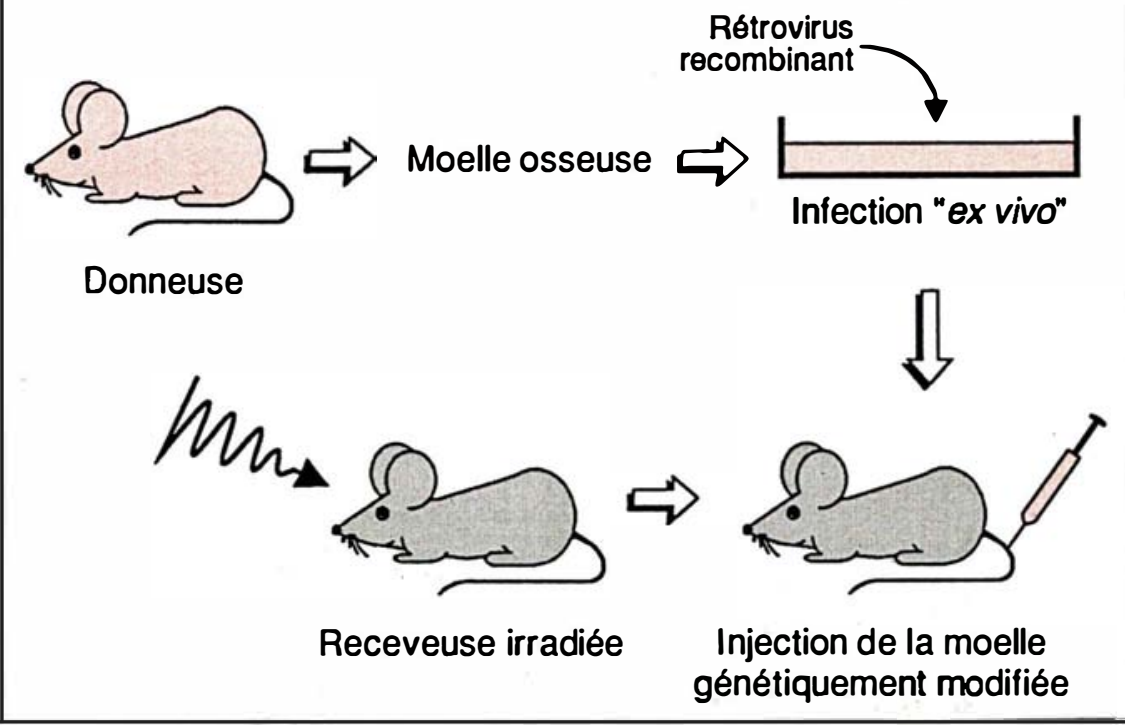

Figure 3. Les diverses étapes du transfert de gènes dans le tissu hématopoïétique in vivo chez la souris. La moelle prélevée est génétiquement modifiée ex vivo puis réinjectée. L'étape d'infection peut aussi consister en une coculture des cellules médullaires avec les fibroblastes producteurs de rétrovirus recombinants. L'animal receveur est habituellement irradié afin de détruire I'hématopoièse endogène et d'obtenir la reconstitution hématologique à partir de la moelle génétiquement manipulée. 
d'envisager la construction de vecteurs $\beta$-globine plus efficaces.

\section{Efficacité de transfert chez la souris in vivo}

Elle dépend beaucoup du titre viral, qui est lui-même fonction de l'efficacité des lignées d'encapsidation et du design du vecteur (figure 3). Elle est donc variable. Lors de l'emploi de stocks viraux de titre élevé, le provirus transféré peut généralement être détecté dans le tissu hématopoïétique de quasiment tous les animaux receveurs à long terme après greffe [30]. Néanmoins, la fréquence du transgène est souvent seulement de l'ordre de 1 cellule " positive" (contenant le provirus) sur 10 [30]. Avec des stocks viraux de titre plus faible, les résultats sont encore moins favorables, seule une faible proportion des animaux receveurs étant alors "positive " [31]. L'efficacité de transfert n'est donc pas encore parfaite. Outre les méthodes déjà mentionnées pour favoriser l'infection des $\mathrm{CSH}$, on peut aussi faire une étape de présélection in vitro avant de réinjecter la moelle manipulée, à condition que le vecteur contienne un gène de sélection (par exemple, une sélection à l'antibiotique G-418 si on utilise un gène néo). Le but est donc, ici, de réinjecter uniquement des cellules ayant effectivement été infectées. On a ainsi pu obtenir jusqu'à $75 \%$ de souris "positives " à long terme dont quasiment toutes les cellules du tissu hématopoiétique possédaient le rétrovirus recombinant [32]. Cependant, une telle étape de présélection $e x$ vivo pourrait être inadéquate pour la thérapie génique, car elle s'accompagne d'une importante perte de cellules souches. En effet, plusieurs souris donneuses sont alors nécessaires pour repeupler une unique souris receveuse. Une sélection in vivo peut aussi être envisagée (voir plus loin).

Le provirus transféré peut servir de marqueur pour des études de filiation cellulaire dans le but de mieux connaître les aspects dynamiques de l'hématopoïèse après greffe. On a ainsi pu prouver qu'il existait réellement des $\mathrm{CSH}$ totipotentes, à longue durée de vie et ayant une haute capacité d'autorenouvellement [17, 18]. De plus, chez la souris, l'héma- topoïèse après greffe semble due à un petit nombre de CSH. Enfin, l'analyse clonale a montré qu'il y avait, durant les premiers mois après la greffe, un recrutement séquentiel des CSH pour assurer l'hématopoïèse et non pas une contribution simultanée de l'ensemble des cellules du pool souche [19]. Plus tardivement après greffe, une hématopoïèse plus stable s'installe, qui semble dépendre de l'émergence et du rôle prépondérant d'un petit nombre de cellules souches topipotentes [33].

\section{Expression du gène transféré chez la souris in vivo}

Les premières études in vivo ont concerné le transfert du gène bactérien néo à l'aide de vecteurs portant le LTR du virus de Moloney. Une faible expression transitoire était détectable, le caractère transitoire pouvant être en rapport avec divers mécanismes : infection préférentielle de précurseurs ou de cellules souches à durée de vie limitée, ou bien dilution ultérieure d'un petit nombre de $\mathrm{CSH}$ infectées au sein d'un nombre plus important de CSH non infectées [17, 20]. Néanmoins, plus récemment, une expression notable du gène néo a pu être obtenue à long terme après autogreffe de cellules médullaires infectées, d'une part, avec un vecteur portant le LTR MPSV et, d'autre part, avec un vecteur comportant le promoteur interne histone $\mathrm{H} 4$ humain [34, 35].

Le transfert d'un $\mathrm{ADN}_{\mathrm{c}}$ codant pour une dihydrofolate réductase mutée $\left(\mathrm{DHFR}^{*}\right)$ (l'astérisque située à droite du signe $D H F R$ signifie qu'il s'agit d'une enzyme mutée) résistante au méthotrexate (MTX) pourrait permettre une sélection (par le MTX) in vivo. L'expression du gène $D H F R^{*}$ transféré a été montrée de façon indirecte. En effet, à l'opposé des souris témoins, des souris greffées avec de la moelle infectée par le vecteur

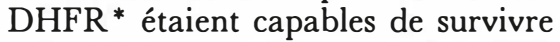
à l'administration précoce de MTX après greffe [36]. Cependant, la survie des animaux ne semble pas due à une véritable sélection, l'administration de MTX n'entraînant pas d'augmentation de la proportion de cellules hématopoïétiques transdui- tes [37]. Avec un vecteur à LTR MPSV, l'expression du gène $D H F R^{*}$ à long terme après greffe a aussi pu être prouvée directement au niveau moléculaire [26].

Récemment, une expression notable d'adénosine déaminase (ADA) humaine a été obtenue de manière stable après greffe. Avec un vecteur comportant un promoteur phosphoglycérate kinase (PGK) humain, on a pu montrer que les progéniteurs hématopoïétiques et les CSH des souris continuaient d'exprimer de l'ADA humaine à long terme [23]. Une importante activité enzymatique ADA a également pu être obtenue dans les diverses lignées hématopoiétiques avec un vecteur comportant le promoteur $\beta$-actine [38]. La question se pose cependant de savoir si le niveau et la répartition de l'expression ADA observée pourraient permettre de reconstituer le système immunitaire d'enfants ayant un déficit en ADA. Un modèle animal (actuellement inexistant) de déficit en ADA trouverait ici son utilité.

A la suite de nombreux travaux in vitro, plusieurs études in vivo ont montré que le gène $\beta$-globine humain transféré par un vecteur génomique s'exprimait spécifiquement au niveau de la lignée rouge à long terme après greffe [28, 31, 32]. Mais le niveau d'expression observé est en général faible, de l'ordre de quelques \% seulement de l'expression de la $\beta$-globine endogène. L'étude de rares thalassémies $\gamma-\delta$ - $\beta$ (où le gène de structure $\beta$-globine est intact mais inactif en raison d'importantes délétions situées loin du gène de structure) a permis de mettre en évidence l'existence des séquences DCR (dominant control region) ou LAR (locus activating region), situées très loin du gène de structure, mais cependant très importantes pour le contrôle de l'expression $\beta$-globine $\left(\mathrm{m} / \mathrm{s} n^{\circ} 4\right.$, vol. 4, p. 452) [39]. Un minilocus $\beta$-globine humaine, comportant ces séquences DCR en plus du gène de structure, est exprimé à un niveau identique à la $\beta$-globine endogène chez des souris transgéniques $\left(\mathrm{m} / \mathrm{s} n^{\circ} 9\right.$, vol. 5, p. 694 et $n^{\circ} 3$, vol. 6, p. 314) [40]. A présent, les recherches visent à disséquer ces longues séquences DCR dans l'espoir de pouvoir inclure leurs éléments essentiels dans un vecteur rétroviral, qui 
RÉFÉRENCES

37. Corey CA, De Silva AD, Holland CA, Williams DA. Serial transplantation of methotrexate-resistant bone marrow : protection of murine recipients from drug toxicity by progeny of transduced stem cells. Blood 1990 ; 75 : 337-43.

38. Wilson JM, Danos O, Grossman M, Raulet DH, Mulligan RC. Expression of human adenosine deaminase in mice reconstituted with retrovirus-transduced hematopoietic stem cells. Proc Natl Acad Sci USA $1990 ; 75: 337-43$

39. Forrester WC, Novak U, Gelinas R, Groudine M. Molecular analysis of the human $\beta$-globin locus activation region. Proc Natl Acad Sci USA 1989 ; 86 : 5439-43.

40. Grosveld F, Van Assendelft GB, Greaves $D R$, Kollias G. Position-independent, high-level expression of the human $\beta$-globin gene in transgenic mice. Cell $1987 ; 51$ : 975-85.

41. Tuan DYH, Solomon WB, London IM, Lee DP. An erythroïd-specific, developmental stage-independent enhancer far upstream of the human " $\beta$-like globin" genes. Proc Natl Acad Sci USA 1989; 86 : 2554-8.

42. Stead RB, Kwok WW, Storb R, Miller AD. Canine model for gene therapy: inefficient gene expression in dogs reconstituted with autologous marrow infected with retroviral vectors. Blood $1988 ; 71$ : 742-7.

43. Kantoff PW, Gillio AP, McLachlin JR, et al. Expression of human adenosine deaminase in non-human primates after retrovirus-mediated gene transfer. $J$ Exp Med $1987 ; 166: 219-34$

44. Parkman R. The application of bone marrow transplantation to the treatment of genetic diseases. Science 1986 ; 232 : 1373-7.

45. Guild BC, Mulligan RC, Gros P, Housman DE. Retroviral transfer of a murine cDNA for multidrug resistance confers pleiotropic drug resistance to cells without prior drug selection. Proc Natl Acad Sci USA 1988 ; 85 : 1595-9.

46. Kasid A, Morecki S, Aebersold P, et al. Human gene transfer: characterization of human tumor-infiltrating lymphocytes as vehicles for retroviral-mediated gene transfer in man. Proc Natl Acad Sci USA 1990 ; $87: 473-77$

47. Miller AD. Communication lors de l'atelier INSERM “Vecteurs rétroviraux pour le transfert de gènes dans les cellules eucaryotes ". Darlix JL, Lehn PM, WeberBenarous A, organisateurs. Paris, 1990.

48. Rosenberg SA, Blaese M, Aebersold P, et al. Immunotherapy of cancer using genemodified tumor infiltrating lymphocytes. devrait donc être capable de transférer un minilocus $\beta$-globine totalement fonctionnel [41]. La correction du phénotype anormal de souris thalassémiques devrait alors devenir possible.

\section{Études in vivo chez de grands animaux}

Les résultats des autogreffes génétiquement modifiées faites chez de grands animaux (chiens, singes) ont été très décevants jusqu'à ce jour. En effet, seulement un très faible niveau d'expression transitoire a pu être détecté, aussi bien après transfert d'un gène $D H F R^{*}$ chez le chien, que d'un gène $A D A$ chez le singe $[42,43]$. On a cependant pu infecter efficacement des progéniteurs hématopoïétiques de diverses espèces (chien, chat, singe, mouton) y compris l'homme [20, 21]. Il devrait donc être également possible de transférer un gène dans les $\mathrm{CSH}$ humaines, mais de nombreuses difficultés devront d'abord être surmontées, notamment en ce qui concerne les conditions d'infection $e x$ vivo des $\mathrm{CSH}$ humaines.

\section{Perspectives de thérapie génique chez l'homme}

Les indications potentielles de thérapie génique dans le cas des maladies héréditaires du sang peuvent se discuter en fonction des résultats obtenus avec les allogreffes de moelle osseuse [1, 2, 44]. En effet, les allogreffes aboutissent également à la mise en place d'un tissu hématopoïétique génétiquement normal. Cependant, les allogreffes nécessitent le recours à un donneur et exposent le receveur à la réaction du greffon contre l'hôte $(\mathrm{GvH})$ dont l'intensité est fonction du degré de compatibilité HLA entre donneur et receveur. Une autogreffe de moelle génétiquement manipulée n'aurait pas tous ces inconvénients et pourrait également constituer un traitement définitif. Dans un premier temps, elle pourrait donc être proposée lorsqu'il n'y a pas de donneur HLA identique dans la fratrie. Les maladies concernées sont les hémoglobinopathies (notamment les thalassémies) et les déficits immunitaires congénitaux (par déficit en ADA). Le rôle du transfert de gènes pour des maladies métaboliques (maladie de Lesch-Nyhan, maladie de Gaucher, maladie de MaroteauxLamy et autres mucopolysaccharidoses...) est souvent plus difficile à apprécier au vu des résultats des allogreffes de moelle osseuse, surtout en ce qui concerne les éventuels effets bénéfiques sur des lésions du système nerveux central [1, 2, 44].

Le transfert de gènes pourrait aussi se révéler utile en cancérologie [1, 2]. On pourrait ainsi envisager de transférer des gènes de résistance à des substances utilisées en chimiothérapie (par exemple, le gène $M D R$ impliqué dans la résistance pléiotropique) [45]. En effet, la possibilité de rendre la moelle osseuse "résistante " pourrait permettre de modifier les protocoles de chimiothérapie et peut-être d'augmenter leur efficacité antitumorale. Il pourrait s'agir d'une allogreffe rendue " résistante " dans le cas des leucémies ou d'une autogreffe "résistante " dans le cas des tumeurs solides sans envahissement médullaire. On peut aussi envisager de modifier génétiquement le système immunitaire de patients cancéreux dans le but de le rendre plus "agressif " à l'égard de la tumeur. Les cellules cibles sont ici des lymphocytes, dont le maniement est peut-être plus aisé que celui des CSH. C'est d'ailleurs pour étudier le devenir à long terme de cellules TIL (tumor infiltrating lymphocytes) utilisées dans un protocole d'immunothérapie, que les Drs Anderson, Blaese et Rosenberg du NIH ont eu l'autorisation d'utiliser un vecteur rétroviral chez des cancéreux au stade terminal. Le gène bactérien néo transféré sert ici tout simplement de marqueur des TIL [46]. Les observations préliminaires ont permis de montrer la persistance des TIL modifiées longtemps après leur réinjection et n'ont pas mis en évidence de problèmes aigus liés à l'emploi de rétrovirus comme vecteurs [47, 48]. Poursuivant l'approche "lymphocytes ", le même groupe du NIH se propose à présent de transférer le gène $A D A$ chez des malades ayant un déficit en ADA.

\section{Conclusion}

L'obtention d'un résultat " physiologique " dans un modèle animal d'une maladie humaine ouvrirait très clai- 
rement la voie à l'utilisation clinique du transfert de gènes au moyen de vecteurs rétroviraux. Cependant, la recombinaison homologue, qui a l'avantage de cibler correctement l'intégration du gène transféré, constitue également une approche intéressante pour la thérapie génique.

De toute façon, quelle que soit la technologie finalement employée, le progrès scientifique devrait permettre, dans un futur plus ou moins proche, le passage d'une génétique moléculaire diagnostique à une génétique moléculaire thérapeutique

\section{Summary}

Retroviral-mediated gene transfer into the hematopoietic tissue in vivo

The use of recombinant retroviruses for gene transfer into the hematopoietic tissue in vivo is still a new area of research. The initial results of in vitro studies were very exciting. In contrast, the early in vivo studies in mice were somewhat disappointing because of the transient and low levels of expression of the transferred gene. Recently, however, better results have been obtained in the murine system in vivo. New packaging cell lines have been constructed, which are safer and still efficient. Better vectors have been designed. Thus, significant levels of expression of the transgene have been achieved in murine long-term transplant recipients. However, the results obtained so far in large animal studies are still disappointing. It should be emphasized that further progress must be based on a simple overall strategy involving better understanding of the functioning of the gene to be transferred, design of vectors carrying a fully active and correctly regulated minilocus and better knowledge of the biological properties of the target cells, the hematopoietic stem cells.

\section{TIRÉS A PART}

P. Lehn. 